\title{
Stability assessment of tunnel face in a layered soil using upper bound theorem of limit analysis
}

\author{
Nima Khezri ${ }^{1}$, Hisham Mohamad ${ }^{* 2}$ and Behzad Fatahi ${ }^{3}$ \\ ${ }^{1}$ UTM Construction Research Centre (UTM CRC), Block C09, Level 1, Institute of Smart Infrastructure and \\ Innovative Construction, Universiti Teknologi Malaysia, 81310, Skudai, Johor, Malaysia \\ ${ }^{2}$ Civil and Environmental Engineering Department, Universiti Teknologi PETRONAS, \\ Bandar Seri Iskandar, 32610 Perak, Malaysia \\ ${ }^{3}$ School of Civil and Environmental Engineering, University of Technology, Sydney, Australia
}

(Received April 02, 2015, Revised March 31, 2016, Accepted May 17, 2016)

\begin{abstract}
Underground tunnelling is one of the sustainable construction methods which can facilitate the increasing passenger transportation in the urban areas and benefit the community in the long term. Tunnelling in various ground conditions requires careful consideration of the stability factor. This paper investigates three dimensional stability of a shallow circular tunnel in a layered soil. Upper bound theorem of limit analysis was utilised to solve the tunnel face stability problem. A three dimensional kinematic admissible failure mechanism was improved to model a layered soil and limiting assumptions of the previous studies were resolved. The study includes calculation of the minimum support pressure acting on the face of the excavation in closed-face excavations. The effects of the characteristics of the layers on the minimum support pressure were examined. It was found that the ratio of the thickness of cover layers particularly when a weak layer is overlying a stronger layer, has the most significant influence on the minimum tunnel support pressure. Comparisons have been made with the results of the numerical modelling using FLAC3D software. Results of the current study were in a remarkable agreement with those of numerical modelling.
\end{abstract}

Keywords: minimum support pressure; stability; layered soil; upper bound; tunnel

\section{Introduction}

Tunnels and underground excavations are one of the important infrastructures in any country. Due to ever increasing growth of the population in the urban areas, tunnelling is seen to be one of the methods to facilitate transportation. Ground settlement and tunnel face collapse are the major concerns in the tunnel design and construction. The settlement of the ground should be within the tolerable limits of the structures above the ground as well as the substructures lying underneath (Mazek 2014). This study investigates the stability of the tunnel face in a layered soil using the upper bound theorem of the limit analysis. The study includes calculation of the minimum support pressure acting on the face of the tunnel in closed-face excavations. The study is limited to the excavation under the compressed air support yet gives some useful information about tunnelling under Earth Pressure Balance (EPB) condition. In excavation under compressed air pressure, the

*Corresponding author, Associate Professor, E-mail: hisham.mohamad@utp.edu.my 
support pressure is uniformly applied to the face of the tunnel to maintain the stability of the face (e.g., Ding et al. 2013, Hassanpour et al. 2009). The paper only considers the collapse of the tunnel face which is triggered by the movement of the soil towards the inside of the tunnel. It is assumed that the tunnel is driven in a cohesive-frictional material.

Laboratory tests, limit equilibrium and limit analysis are some of the major approaches which have been used by researchers to investigate the face stability of shallow tunnels in cohesivefrictional soils (with significant or insignificant cohesion). Chambon and Corté (1994) conducted some centrifuge tests on cohesionless soils. The aim of centrifuge tests of Chambon and Corté (1994) was to study the pattern of soil failure in tunnels and also to estimate the critical support pressure. Failure mechanism and stability of tunnel face were investigated by Takano et al. (2006). They conducted some model tests using an X-Ray computed tomography scanner to obtain a 3D visualization of the failure zone. Their results show that the failure mechanism is like a logarithm spiral curve in longitudinal section and can be simulated as an ellipse in horizontal cross section.

Studies of tunnel face stability concerning limit equilibrium method mostly rely on the silo theory of Jannsen (1895). The first adoption of this theoretical approach was made by Horn (1961), proposing the wedge stability model. Horn's (1961) model divided the ground into two rigid blocks where tunnel's circular area was approximated by a rectangle. Engineering application of Horn's (1961) wedge stability model was presented by Jancsecz and Steiner (1994). Jancsecz and Steiner (1994) calculated the support pressure to balance water and earth pressure at the face of the tunnel. Their model also considers the heterogeneity of the soil above the tunnel face. Anagnostou and Kovari (1994) implemented the Horn's (1961) model and studied the infiltration of the slurry on the face of the excavation. In coarse material and over the time, the slurry will infiltrate into the face. Later on, Anagnostou and Kovari (1996) used wedge model to analyse the face stability problem in drained condition. Their study is limited to the machine operation in the EPB and their results show the relationship between the limit pressure and hydraulic head in the muck. Broere (1998) used wedge stability model to study the effect of the soil heterogeneity in front of the tunnel face on the face stability. The missing physics in limit equilibrium method is that they do not consider the stress strain relationship and consequently do not ensure displacement compatibility. This may result in a conservative and overestimated support pressure which will be described later.

One of the interesting methods available to estimate the support pressure of shallow tunnels belongs to Leca and Dormieux (1990). Their three-dimensional mechanisms consist of conical blocks. The two conical mechanism of Leca and Dormieux (1990) for collapse is shown in Fig. 1. In this mechanism, the second block (Block OFB) is the mirror image of the Block OBD with respect to plane CG in a way that Block OFB is always vertical. CG is the bisector perpendicular of $\mathrm{OB}$. Because of the verticality assumption, the mechanism suffers from lack of degree of freedom as only one block (Block OBA in Fig. 1) is allowed to move in optimization process. Mollon et al. (2009) increased the number of rigid blocks involved in the collapse mechanism and resolved the limiting assumption for two conical block mechanism (verticality of the second block). The whole circular face of the tunnel was encountered in the support pressure calculations of Mollon et al. (2010). Spatial discretization technique made it possible to generate the three dimensional failure surface point-by-point instead of using standard geometrical shapes such as cones. Their results for frictional and cohesive soil were presented in the form of design charts. Tang et al. (2014) improved the two conical mechanism (Fig. 1) of Leca and Dormieux (1990) to be applicable in a layered soil. However, the aforementioned deficiency of the verticality of the second block in their solution remains unresolved. More recently, Senent and Jimenez (2015) 


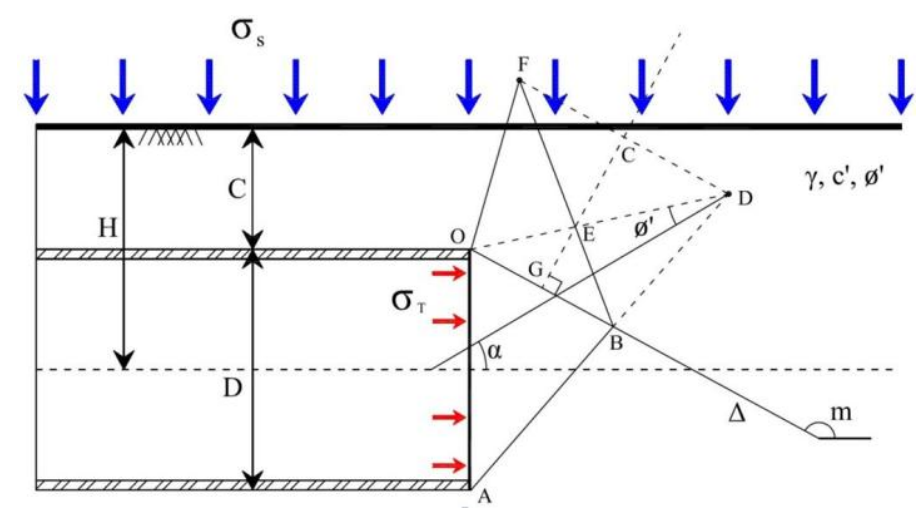

Fig. 1 Two conical mechanism of Leca and Dormieux (1990)

modified Mollon et al. (2011) rotational failure mechanism to investigate the critical collapse pressure in addition to the partial failure of tunnel face when a weak layer overlying a stiff layer. Ibrahim et al. (2015) also extended rotational failure mechanism developed by Mollon et al. (2011) to investigate the minimum support pressure in stratified ground, but their work is limited to purely frictional materials.

In addition, Leca and Dormieux (1990) compared their results with the centrifuge tests of Chambon and Corté (1989). The results of upper bound were in a good agreement with the actual measurements of the centrifuge tests. Kirsch (2010) conducted some 1-g physical tests and compared the outcomes of the tests with available analytical solutions. It turned out that Leca and Dormieux (1990) solution predicts the support pressure with high levels of accuracy. The simplicity and accuracy (to some extent) of Leca and Dormieux (1990) method keeps it popular and it is the base of failure mechanisms used in the current research (e.g., Khezri et al. 2015). This article provides an improved solution to estimate the minimum support pressure of the shallow circular tunnels in a layered soil. Two conical mechanism of Leca and Dormieux (1990) has been modified to model a potential failure in a layered soil. Thus the aforementioned limiting assumption of Leca and Dormieux (1990) (verticality of second block) has been resolved. Employing these improved mechanisms, a parametric study has been conducted to study the influence of various soil and geometry parameters on the stability of the tunnel face.

\section{Problem definition and upper bound analysis}

Fig. 2 shows two shallow circular tunnels in two types of layered soil. The tunnel diameter is $D$ and the total cover of the tunnel is $C=C_{1}+C_{2}$. The depth ratio is defined as $C / D$. The annotation in these figures is defined herein: index " 1 " refers to the upper layer and index "2" refers to the layer which contains the tunnel. The layer containing the tunnel is called the crossed layer and the distance between the tunnel crown and the ground surface is called the cover. The boundary of the layers is located in between $\left(C_{1}\right.$ and $\left.C_{2}>0\right)$ or on the tunnel crown $\left(C_{2}=0\right)$ and the ground surface $\left(C_{1}=0\right)$. Soil unit weights for layers 1 and 2 are $\gamma_{1}$ and $\gamma_{2}$, respectively. A uniform vertical surcharge of $\sigma_{s}$ can be considered on the ground surface. Tunnel face is allowed to move while a uniform pressure of $\sigma_{T}$ is applied on the face of the tunnel. Soil layers are modelled using MohrCoulomb material with cohesions of $c_{1}, c_{2}$ and internal friction angles of $\phi_{1}, \phi_{2}$. The collapse 


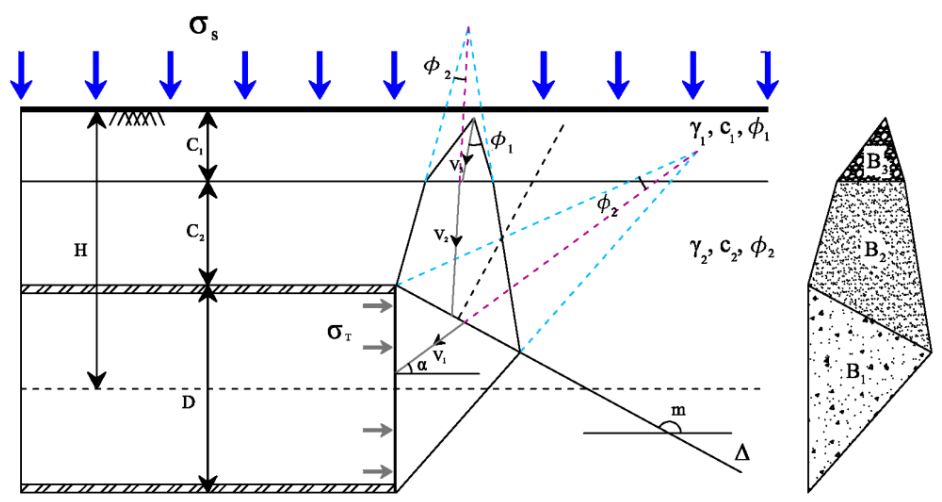

(a) $\phi_{1}>\phi_{2}$

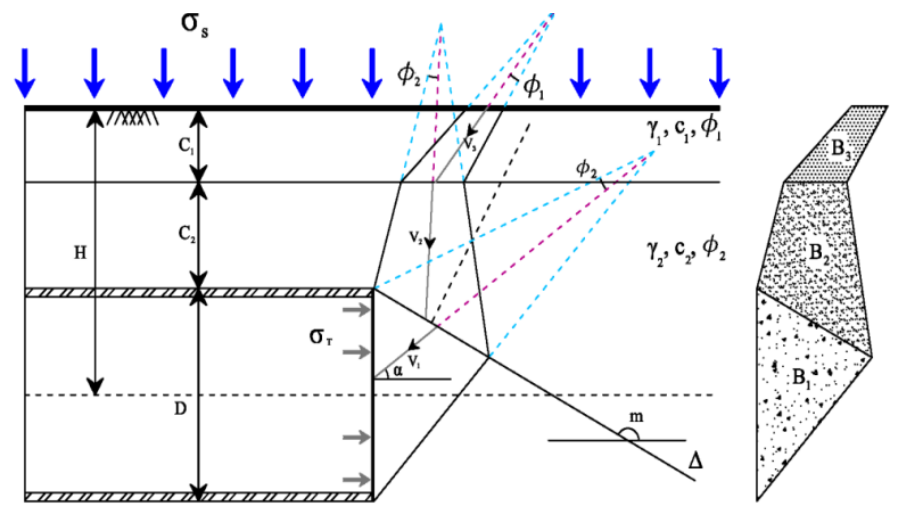

(a) $\phi_{1}<\phi_{2}$

Fig. 2 Failure mechanism for a soil stratum with two layers while

mechanisms consist of three rigid blocks $B_{1}, B_{2}, B_{3}$. Each block is a part of its generatrix cone with apex angle of $2 \phi_{i}(i=1,2)$ in order to satisfy the normality condition which is truncated by several planes like the tunnel face, plane $\Delta$, boundary of layers and the ground surface. The derivations related to the mechanisms in Fig. 2 and also the optimisation procedure are given in Appendix A.

\section{Face stability analysis}

\subsection{Comparison with previous solutions}

Tang et al. (2014) modelled a fixed diameter of shallow tunnel to conduct a parametric study in the layered soil. The same example was employed here as a comparison tool. The tunnel diameter $D$ is $6 \mathrm{~m}$. It is assumed that the tunnel cover $C$ consists of only one layer and the layer's boundary passes through the tunnel crown. The soil in layers is homogeneous and incompressible. Considering a single cover layer makes the $C_{2}$ in Fig. 2 equal to zero. This assumption does not affect the validity of the solution as in the previous section, it was mentioned that the layer's boundary could locate at any position from tunnel crown to the ground surface. It should be mentioned that the results of the current study and Tang et al. (2014) have been compared with 
Broere (2001). Broere (2001) has shown that a wedge stability model based on limit equilibrium can also be used to determine the minimum support pressure in EPB shield tunnelling to prevent a face collapse.

\subsubsection{The effect of strength parameters of the crossed layer on the minimum support pressure}

The soil unit weight for both layers are assumed to be $\gamma_{i}=18 \mathrm{kN} / \mathrm{m}^{3}, i=1,2$. The shear strength of the cover layer (upper layer) was kept constant while the shear strength of the crossed layer (lower layer containing tunnel) was varied gradually and its effect on the minimum support pressure was studied. The cohesion of the cover layer $c_{1}=2.5 \mathrm{kPa}$ and the internal friction angle of the cover layer $\phi_{1}=20^{\circ}$ were adopted. $\Delta c_{2}$ is introduced as the difference of the cohesion values of the crossed and cover layers. The cohesion of the crossed layer is defined by $c_{2}=c_{1}+\Delta c_{2}$. Considering $\phi_{1}$ and $\phi_{2}$ as the friction angles of the cover and crossed layers, respectively, $\Delta \phi_{2}$ can be defined as the difference of the friction angles of the crossed and cover layers $\phi_{2}=\phi_{1}+\Delta \phi_{2}$. No surcharge is assumed to act on the ground surface $\left(\sigma_{s}=0 \mathrm{kPa}\right)$.

Fig. 3 shows the influence of $\Delta \phi_{2}$ on the minimum support pressure, $\sigma_{T}$. As Tang et al. (2014) predicted, increasing the friction angle of the crossed layer results in the decrease of the minimum support pressure. This descending trend is steeper in the range of -15 to 0 for $\Delta \phi_{2}$. The use of present failure mechanism results in higher upper bound tunnel support pressures than available

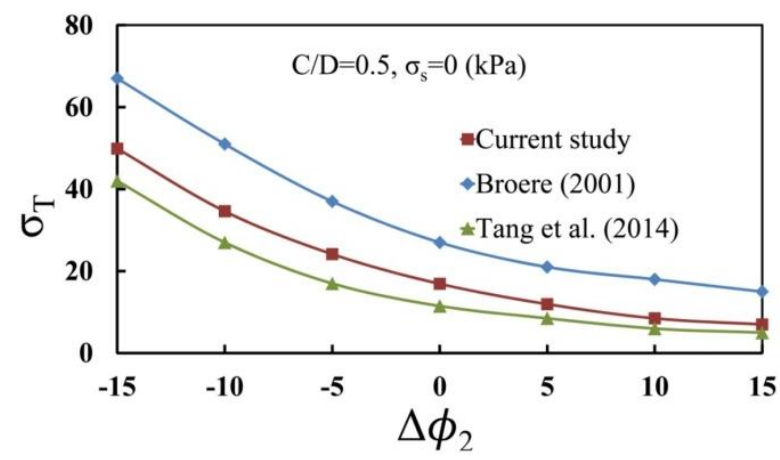

Fig. 3 Effect of variation of crossed layer's friction angle on the minimum support pressure

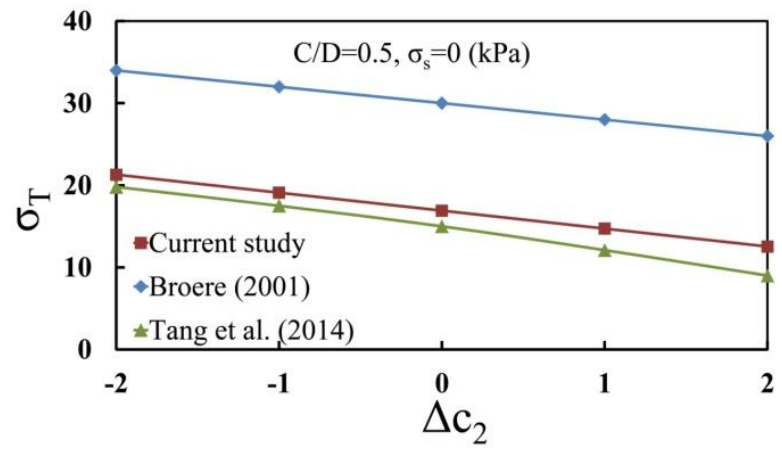

Fig. 4 Influence of increase in crossed layer's cohesion on the minimum support pressure $\left(\Delta \phi_{2}=0\right)$ 
solution of Tang et al. (2014) with an average improvement of $38.16 \%$ for the aforementioned example.

Fig. 4 shows the influence of $\Delta c_{2}$ on $\sigma_{T}$. Cohesion of the crossed layer was increased in contrast to the cover layer's cohesion while the cohesion of the cover layer was kept constant. Other soil parameters for both layers were assumed to be the same. A steady descending trend of tunnel support pressure, against the increase of the $\Delta c_{2}$ is observed in Fig. 4. However the current study yields higher upper bound of the $\sigma_{T}$ than the available solution of Tang et al. (2014). The average improvement of the minimum support pressure is $18.1 \%$.

Comparative study on both strength parameters of $\Delta c_{2}$ and $\Delta \phi_{2}$ (Figs. 3 and 4) shows that the current solution results greater upper bound minimum support pressure predictions than previous available solution of Tang et al. (2014). As mentioned earlier, the collapse mechanisms of Leca and Dormieux (1990) and subsequently Tang et al. (2014) does not offer that much of degree of freedom. Because of the slight change in the shape of the failure mechanism in the current study, the support pressure resulted from current solution seems to be improved in comparison to the previous study. On the other hand, Limit Equilibrium wedge model of Broere (2001) as expected results in greater values of the minimum support pressure than the upper bound solutions.

\subsubsection{The effect of strength parameters of the cover layer on the minimum support pressure}

The effect of shear parameters of the cover soil on the minimum support pressure is investigated in this sub-section. The boundary of the crossed soil and the cover soil is assumed to be adjacent to the tunnel crown. The soil properties of the crossed soil are $\gamma_{2}=18 \mathrm{kN} / \mathrm{m}^{3}, \phi_{2}=20^{\circ}$ and $c_{2}=2.5 \mathrm{kPa}$. The difference between the cohesion of the cover layer and the cohesion of the crossed layer is $\Delta c_{1}=c_{1}-c_{2}$. Similarly the difference between the friction angles of the cover layer and the crossed layer is $\Delta \phi_{1}=\phi_{1}-\phi_{2}$. To simplify the procedure, the effect of surcharge is not taken into account here. Fig. 5 shows the variation of $\sigma_{T}$ against the subtraction of friction angles of the cover layer and the crossed layer $\left(\Delta \phi_{l}\right)$ while the friction angle of the crossed layer is kept constant. The curves obtained from current solution (solid lines) show nonlinear decrease of minimum support pressure. Moreover, the current solution yields higher upper bounds than the previous solution (dotted lines) with an average improvement of tunnel support pressure of $12.24 \%$ for the domain of $-15<\Delta \phi_{1}<15$ and $C / D=0.5$.

Fig. 6 shows the estimated minimum support pressure plotted against the variation of $\Delta c_{1}$, that is, the cohesion of the cover layer varies while the cohesion of the crossed layer is kept constant.

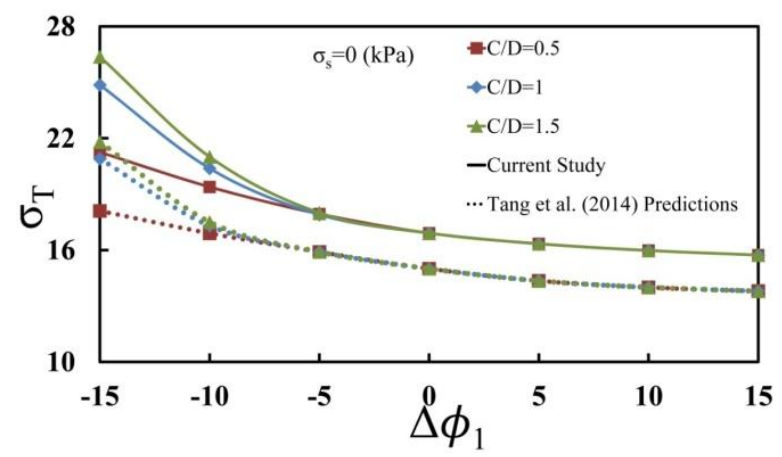

Fig. 5 Effect of varying the friction angle of the cover layer on the minimum support pressure 


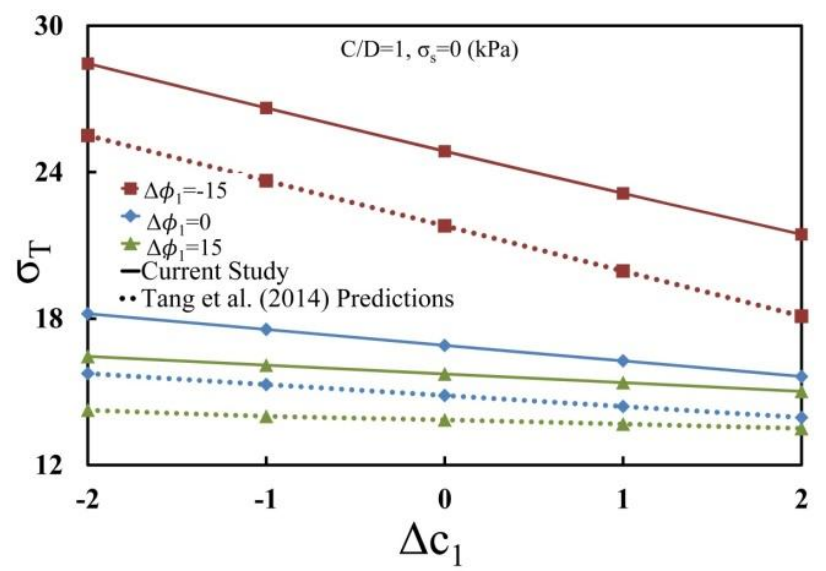

Fig. 6 Effect of varying cohesion of the cover layer on the minimum support pressure

Improvements to the previous solution are substantial. For $\Delta \phi_{1}=15$, the results of the current upper bound solution are 14.8\% greater than Tang et al. (2014) predictions (see red lines in Fig. 6).

\subsection{Comparison with FLAC3D results}

Finite difference software FLAC3D (Fast Lagrangian Analysis of Continua) has been used to perform the numerical simulation (Itasca Consulting Group 1993). The internal programming capability of the software (FISH) allows addition of user defined subroutines. The stability of a system in FLAC3D simulations is defined by unbalanced force ratio, that is, the average unbalanced mechanical force divided by the average applied mechanical force at all the grid points of the system. A system is considered at the state of the static equilibrium when the unbalanced force ratio is less than the tolerance value $10^{-5}$ recommended by Itasca Consulting Group (1993).

In order to conduct all the calculations, the following procedure is performed. Firstly, according to the input data, the geometry of the soil body is constructed and then the geostatic body forces are applied to the domain (Fig. 7). Secondly, the displacement of all points of the body is set to zero as only the displacements due to support pressure are applied here.

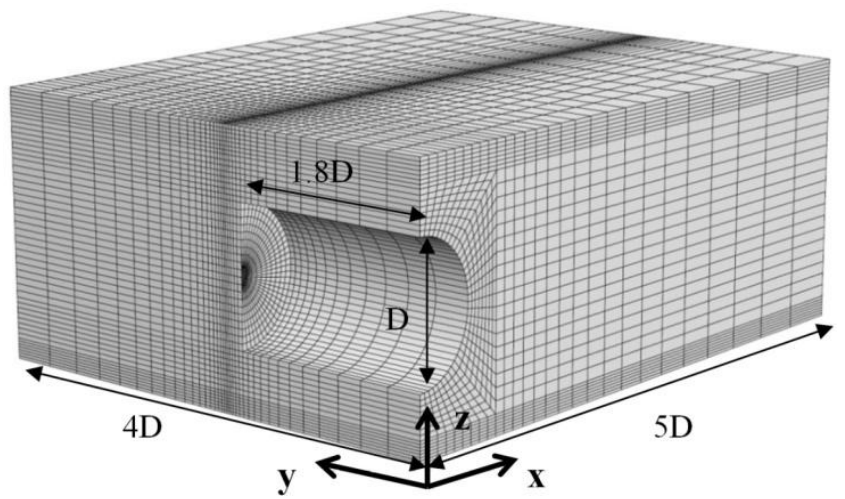

Fig. 7 Body of soil domain and zone configuration used in FLAC3D 
Considering Fig. 7 and the direction of the axis, the numerical model has the size of $5 D$ in $x$ direction, $4 D$ in y-direction and $1.3 D+1.3 \mathrm{C}$ in $z$ direction. These dimensions are chosen in a way that the boundary conditions do not affect the support pressure or the safety factor calculations. The length of the tunnel in $y$-direction is $1.8 D$. These boundaries are suggested in Mollon et al. (2009) to be enough not to alter the distribution of the stresses. A non-uniform three-dimensional mesh was chosen for this problem. The model consists of 48600 zones and 51837 grids. The density of mesh has been increased in locations where high stress gradients were expected. All the movements at the bottom face of the model are fixed while the ground surface is free to move. Vertical boundaries at the sides of the soil box are only fixed in the direction normal to their surface (only horizontal displacement is fixed by use of roller boundaries).

Mohr-Coulomb failure criterion is chosen to model the soil. The elastic properties of the soil are Young's modulus $E=240 \mathrm{MPa}$ and Poisons ratio $v=0.3$. It should be mentioned that the soil elastic parameters do not have significant effects on this type of stability analysis. Considering the altitude of the layers, material groups are assigned to the respective layers. A concrete tunnel lining with thickness of $0.4 \mathrm{~m}$ is created by use of shell elements. Elastic properties of the lining are $E=$ $15 \mathrm{GPa}$ and $v=0.2$. Interface elements which follow Coulomb's law are employed to connect the lining to soil elements. The friction angle of the interface is assumed to be two-third of the soil's friction angle. Normal stiffness and shear stiffness of $K_{n}=10^{11} \mathrm{~Pa} / \mathrm{m}$ and $K_{s}=10^{11} \mathrm{~Pa} / \mathrm{m}$ are assigned to the interface, respectively. These parameters are a function of neighbouring elements rigidity (Itasca Consulting Group 1993) and the accuracy of this type of stability analysis will not be affected by these parameters.

The support pressure is determined by use of a bisection method. The bisection method consists of bracketing and bisecting for several trial support pressures. The support pressure is the required minimum support pressure which should be exerted on tunnel face to maintain the stability. In this procedure, it is necessary to establish the upper and lower brackets of the support pressure which correspond to the stable and unstable condition of the tunnel, respectively. To establish the upper bracket of the support pressure, a high value for support pressure (not so high to cause blow-out) should be chosen which will result in stable condition of the model at the end of the model calculation run. Unbalanced force should be monitored during this procedure and as soon as the maximum unbalanced force falls below the prescribed value, it indicates that the model is in state of equilibrium. Any trial value of support pressure which results in steady state of equilibrium can be chosen as the upper bracket of the support pressure. On the other hand, any small magnitude of the support pressure which results in the steady state of plastic flow (unbalanced force reaches to a non-zero constant value) determines the lower bracket of the support pressure. Once the upper and lower brackets are established then a new value for support pressure, midway between the upper and lower bracket, is chosen. The model is tested for the new value of the support pressure and in case it responded a stable condition, the upper bracket would be replaced by the new value. It is obvious that if the new value of the support pressure corresponds to an unstable condition, the lower bracket should be replaced with it. The aforementioned procedure should be repeated several times until the difference between the upper and lower bracket falls in a prescribed tolerance.

A comparison between the results obtained by the current solution and FLAC3D has been made to evaluate the accuracy of the proposed upper bound solution. The example in Section 3.1.1 has been employed here to conduct a numerical simulation. It is assumed that a uniform retaining pressure is applied to the face of the tunnel to simulate the tunnel boring under compressed air condition and lining is installed immediately after the boring and the unlined length of the tunnel 
prior to face is equal to zero. Because of the symmetry, only one half of the model is considered in the analysis as shown in Fig. 7. The soil dilation angle $\psi$ is in accordance with the commonly used formula of $\psi=\phi-30^{\circ}$. In the second stage of the simulation, which is, after generating the mesh and soil body and applying the gravitational forces, a NULL model was assigned to the range of the tunnel. NULL model is one of the internal models of $\mathrm{FLAC}_{3 \mathrm{D}}$ which is used to represent material that is removed or excavated. The shell elements (tunnel lining) were then assigned and the face pressure was applied to the face of the excavation. It is assumed that the tunnel advances $1.8 D$ instantaneously.

Fig. 8 shows the comparison made between the results of the proposed upper bound solution and the numerical simulations. Similar to the results shown in Fig. 3, tunnel support pressure obtained from the numerical simulation decreases non-linearly with the increase of the crossed layer's internal friction angle. Comparing the results of the current study and $\mathrm{FLAC}_{3 \mathrm{D}}$ simulations shows a remarkable agreement between the two methods.

Fig. 9 shows an example of failure mechanism computed by $\mathrm{FLAC}_{3 \mathrm{D}}$ and the upper-bound analyses, both of which indicating the collapse reaches ground surface when $\Delta \phi_{2}=-5$ and for the

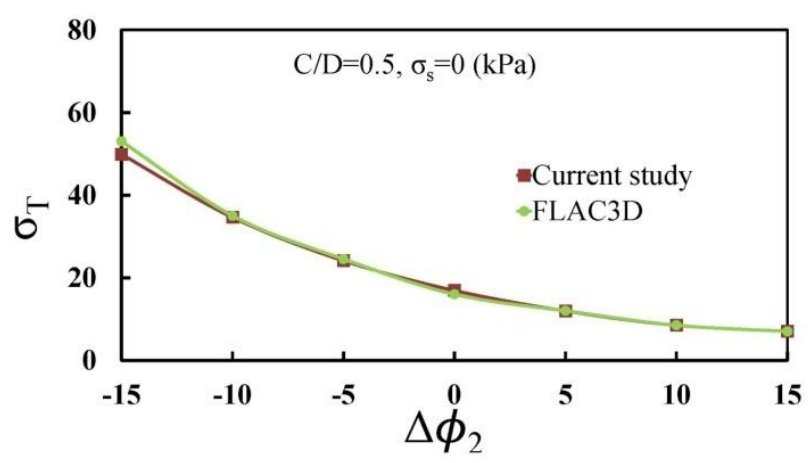

Fig. 8 Comparison of results obtained by current upperbound solution and FLAC3D simulations

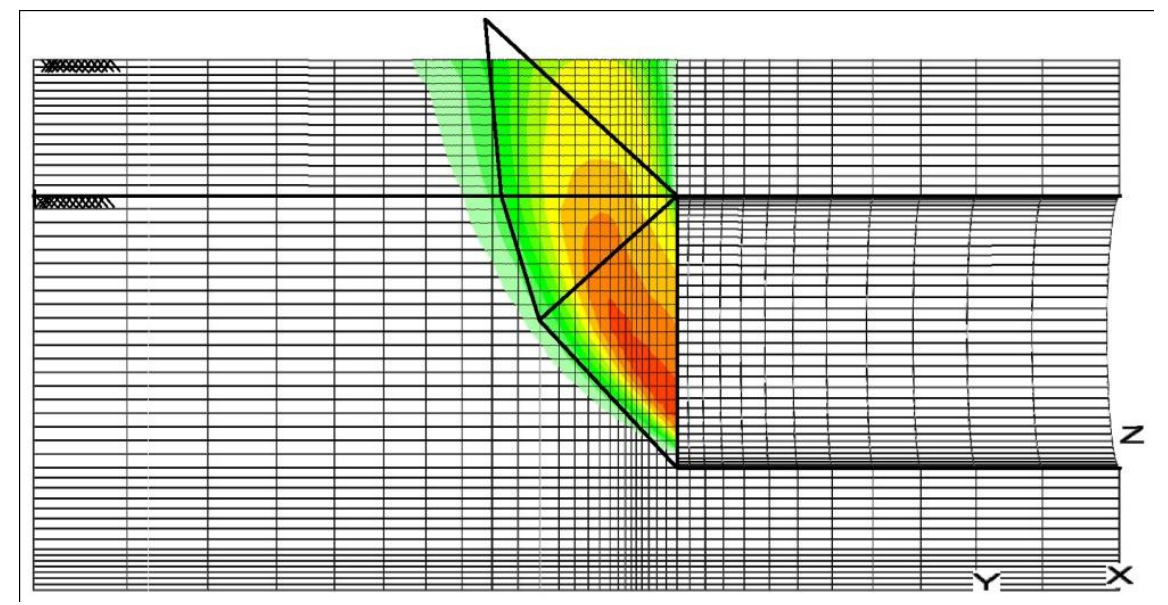

Fig. 9 Comparison of failure mechanisms computed with the limit analysis mechanism and with the numerical model $\left(\Delta \phi_{2}=-5\right)$ 
same soil parameters in Section 3.1.1. In case of the upper-bound method, the final truncated cone is not vertical but situated at an angle of $25^{\circ}$ from vertical for optimum support pressure. This indicates why the proposed upper bound solution is very consistent with the full numerical analysis, and hence is suitable to estimate the minimum support pressure in various two-layered soil system.

\subsection{Influence of tunnel cover on minimum support pressure}

The tunnel and soil characteristics reported in Section 3.1.2 were adopted here to show the variation of the $\sigma_{T}$ against the depth ratio $(C / D)$. Fig. 10 shows that for $\Delta \phi_{1}=-15^{\circ}$ the minimum support pressure increases slightly and afterwards remains constant. For cases of the soil deposits where the cover layer's friction angle is equal or greater than the crossed layer's friction angle, the $\mathrm{C} / \mathrm{D}$ ratio has no effect on the $\sigma_{T}$. However, the minimum support pressure calculated using Broere's Limit Equilibrium method is greatly influenced by the depth of tunnel (Fig. 10) with $\sigma_{T}$ increased almost linearly with the tunnel depth. It should be mentioned that similar trends have been obtained by Tang et al. (2014) to the current solution (not shown in Fig. 9 and the following figures) but with less satisfactory of lower $\sigma_{T}$ values.

\subsection{Influence of thickness of layers $\left(C_{1}>0\right.$ and $\left.C_{2}>0\right)$}

The effect of strength parameters of soil as well as the thickness of each layer is discussed in this section. The tunnel diameter is assumed to be $6 \mathrm{~m}$ and soil unit weight is $18 \mathrm{kN} / \mathrm{m}^{3}$. Fig. 11 shows the minimum support pressure against the thickness ratio $C_{1} / C_{2}$ for three values of $\Delta \phi_{2}$ (the cover layers friction angle is $20^{\circ}$ and constant) for several depth ratios. When $\Delta \phi_{2}<0, \sigma_{T}$ decreases as the thickness of the upper layer increases particularly for depth ratios $C / D>0.5$. However changing the thickness of the layers generally does not have any effect on the minimum support pressure for $\Delta \phi_{2} \geq 0$. One can conclude that when a soil layer with higher value of friction angel is located above a low friction angle soil layer, increasing the thickness of the upper layer with higher friction angel affects the minimum support pressure notably.

Fig. 12 shows how the minimum support pressure varies with layer's thickness ratio for three values of $\Delta c_{2}$. The cohesion of the upper layer is held constant at $2.5 \mathrm{kPa}$. In general, the variation of the thickness of the upper layer in low cohesive soils does not have any significant effect on $\sigma_{T}$.

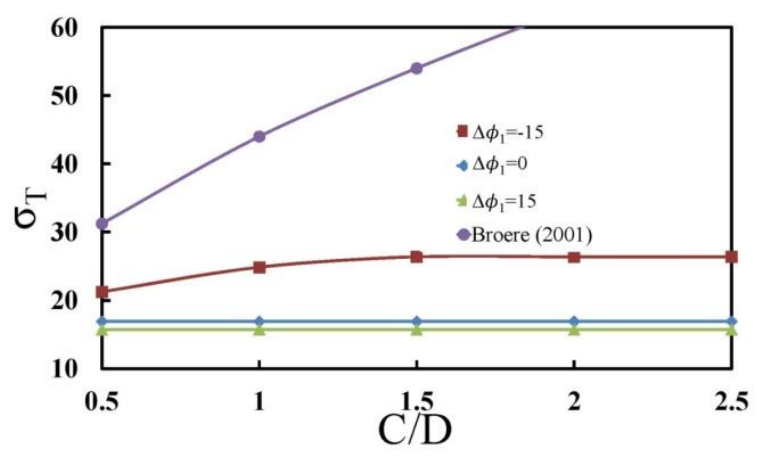

Fig. 10 Influence of tunnel cover on minimum support pressure for different values of cover layer's friction angle 


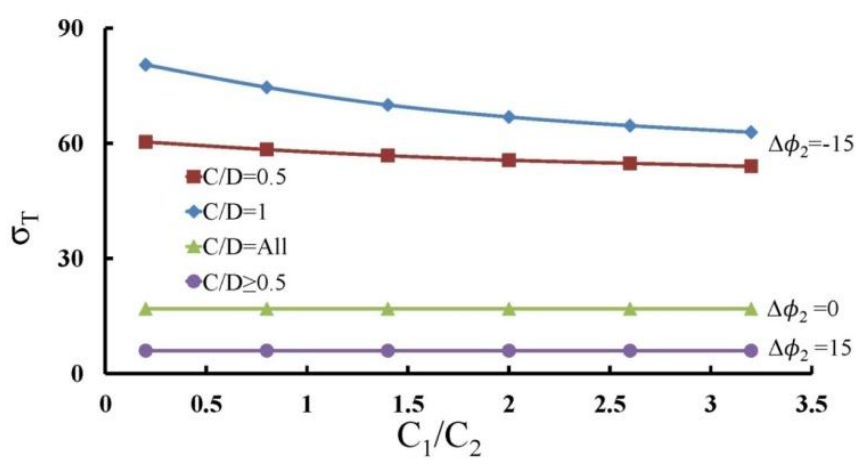

Fig. 11 Minumum support pressure against thickness of layers $\left(\Delta c_{1}\right)$

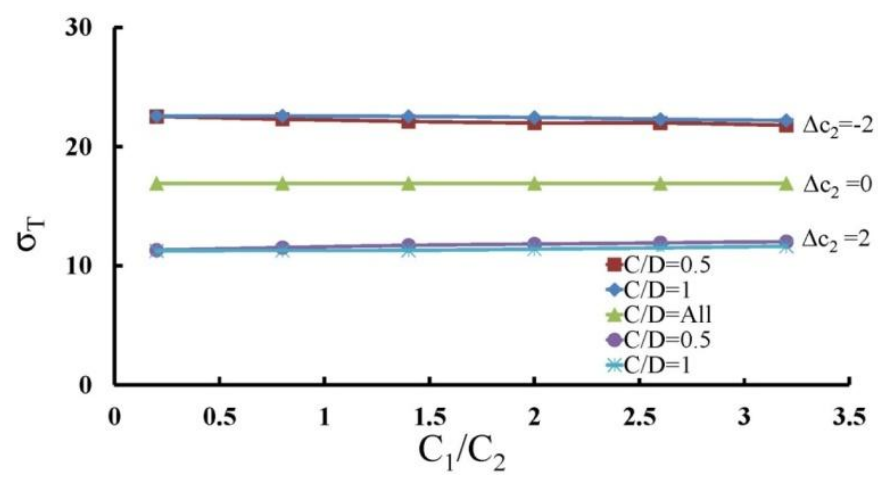

Fig. 12 Minimum support pressure against thickness of layers $\left(\Delta \phi_{1}\right)$

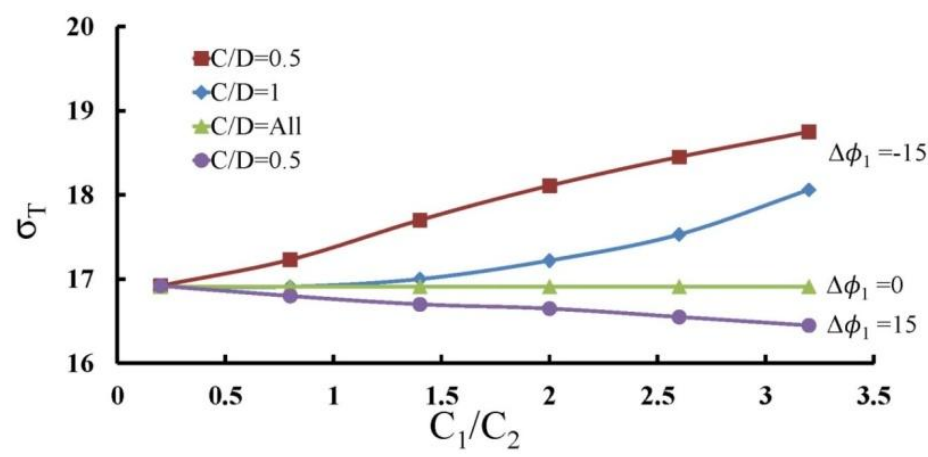

Fig. 13 Minimum support pressure against thickness of layers $\left(\Delta c_{2}=0\right)$

The influence of soil thickness ratio $C_{1} / C_{2}$ on minimum support pressure for three values of $\Delta \phi_{1}$ and depth ratios are presented in Fig. 13. The friction angle of the upper layer is $\phi_{1}=20^{\circ}$ and constant. For soils where $\Delta \phi_{1}<0$ (upper layer with lower friction angle), a slight increase in minimum support pressure is observed while for soils with $\Delta \phi_{1}>0$, minimum support pressure decreases as the thickness of the upper layer increases. As evident in Fig. 13, variation of the thickness of the layers in this context have insignificant impact on minimum support pressure 


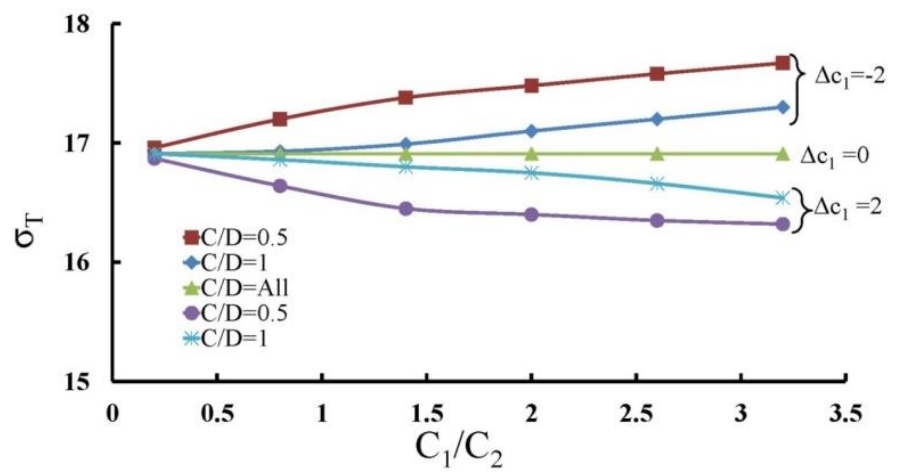

Fig. 14 Minimum support pressure against thickness of layers $\left(\Delta \phi_{2}=0\right)$

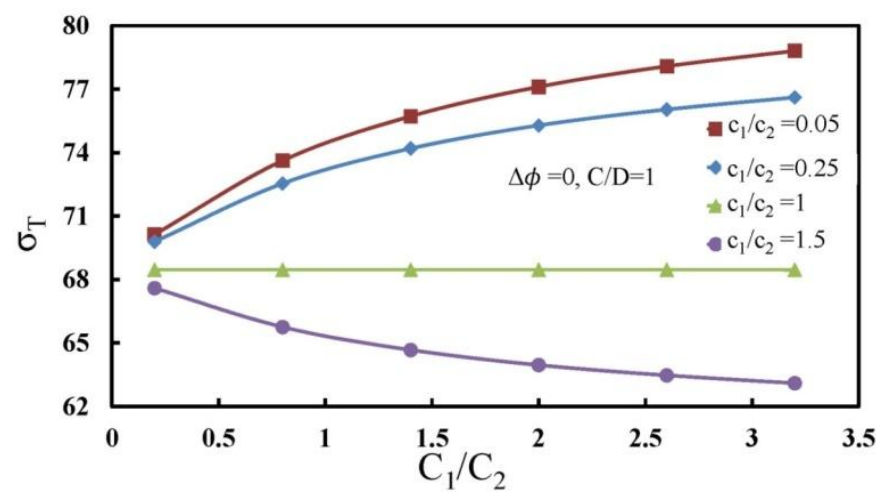

Fig. 15 Effect of layer thickness ratio on the minimum support pressure when cohesive ratio is varied

while $\Delta \phi_{1}$ is considered as the controlling parameter (when the crossed layer's parameters are kept constant).

For cases where the cohesion of the lower layer is kept constant and changes have only been made to the upper layer's cohesion $\left(\Delta c_{1}=\right.$ Var.), small changes in minimum support pressure can be observed, as shown in Fig. 14. These fluctuations however are not significant. Thus it can be inferred that varying the thickness of the layers while the parameters of the lower layer are kept constant $\left(\Delta c_{1}=\right.$ Var., $\left.\Delta \phi_{1}=0\right)$ does not have a significant effect on $\sigma_{T}$.

Fig. 15 shows the variation of the minimum support pressure $\sigma_{T}$ against the thickness ratio of the layers $C_{1} / C_{2}$ (where $C=C_{1}+C_{2}$ ) for four ratios of cohesion of the layers $c_{1} / c_{2}$. Tunnel diameter $D$ is taken as $10 \mathrm{~m}$, soil friction angle in layers $\phi$ is $10^{\circ}$, soil unit weight $\gamma$ equals to 19 $\mathrm{kN} / \mathrm{m}^{3}, c_{2}=7 \mathrm{kPa}$ and $\sigma_{s}=20 \mathrm{kPa}$. As observed, for cases with cohesion ratio $c_{1} / c_{2}$ less than 1 , the $\sigma_{T}$ increases with increasing $C_{1} / C_{2}$ ratios. (Fig. 15 is another representation of Fig. 14 except that the soil friction angle is smaller and the cohesion is greater than those in Fig. 14). In general, reduction of the friction angle of the soil results in taller failure mechanism (the theoretical height of the failure mechanism from bottom of the tunnel to the apex is greater in soils with small friction angel assuming the same tunnel). Increasing the thickness of the upper layer takes the larger portion of the mechanism and consequently the soil parameters in this layer affect the minimum support pressure. Thus, it can be concluded that the ratio of the thickness of cover layers 
has a significant influence on the minimum support pressure depending on the value of friction angles of the soil layers. It should be noted that when a weak layer is overlying a stronger layer, the thickness of the weak layer has significantly influence the magnitude of the minimum support pressure (depending on the internal friction angle and height of the failure mechanism).

\section{Conclusions}

Upper bound theorem of limit analysis has been used to study the stability of tunnel face in a layered soil. The model originally developed by Leca and Dormieux (1990) and modified by Tang et al. (2014) was employed to simulate the stability of tunnel face. The limitations of these approaches were resolved in the current study by searching the optimum angle of second block. A comparative study was conducted to compare the results of the current study and previous solutions. In all the comparisons, the trend of variation of the minimum support pressure was similar to previous study except that the new solution yielded higher upper bounds which may be considered as improvements of the previous solution. On the other hand, results obtained by numerical simulation, using FLAC3D software, show a remarkable agreement with the results of the current study. In addition, the effect of shear strength of the soil layers on the minimum support pressure has been investigated. It was shown that increasing the thickness of the upper soil layer, regardless of being stronger or weaker than the lower layer, affects the minimum support pressure. However, the magnitude and severity of this influence depends on the value of the friction angle. It was shown that variation of minimum support pressure against thickness ratio in low frictional soils is more significant than the high frictional material.

\section{References}

Anagnostou, G. and Kovari, K. (1994), "The face stability of slurry-shield driven tunnels", Tunn. Undergr. Space Technol., 9(2), 165-174.

Anagnostou, G. and Kovari, K. (1996), "Face stability conditions with earth-pressure-balanced shields", Tunn. Undergr. Space Technol., 11(2), 165-173.

Broere, W. (1998), "Face stability calculation for a slurry shield in heterogeneous soft soils", Proceedings of World Tunnel Congress 98 on Tunnels and Metropolises, Volume 1, Balkema, Rotterdam, The Netherlands, pp. 215-218.

Broere, W. (2001), "Tunnel face stability and new CPT applications", Ph.D. Thesis; Geotechnical Laboratory, Delft University of Technology, The Netherlands.

Chambon, P. and Corté, J.F. (1989), "Stabilité du front de taille d'un tunnel faiblement enterré: modélisation en centrifugeuse", Proceedings of the International Conference on Tunnelling and Microtunneling in Soft Ground: From Field to Theory, Paris, France, pp. 307-315.

Chambon, P. and Corté, J.F. (1994), "Shallow tunnels in cohesionless soil: Stability of tunnel face", J. Geotech. Eng., 120(7), 1148-1165.

Ding, W.Q., Peng, Y.C., Yan, Z.G., Shen, B.W., Zhu, H.H. and Wei, X.X. (2013), "Full-scale testing and modeling of the mechanical behavior of shield TBM tunnel joints", Struct. Eng. Mec., Int. J., 45(3), 337354.

Hassanpour, J., Rostami, J., Khamehchiyan, M. and Bruland, A. (2009), "Developing new equations for TBM performance prediction in carbonate-argillaceous rocks: A case history of Nowsood water conveyance tunnel", Geomech. Geoeng.: Int. J., 4(4), 287-297.

Horn, M. (1961), "Horizontal earth pressure on perpendicular tunnel face", Proceedings of the Hungarian National Conference of the Foundation Engineer Industry, Budapest, Hungary, pp. 7-16. [In Hungarian] 
Ibrahim, E., Soubra, A.H., Mollon, G., Raphael, W., Dias, D. and Reda, A. (2015), “Three-dimensional face stability analysis of pressurized tunnels driven in a multilayered purely frictional medium", Tunn. Undergr. Space Technol., 49, 18-34.

Itasca Consulting Group, Inc. (1993), Fast Lagrangian Analysis of Continua, User's Manual.

Jancsecz, S. and Steiner, W. (1994), "Face support for a large mix-shield in heterogenous ground conditions”, In: Tunneling ‘94, Springer US, pp. 531-550.

Janssen, H.A. (1895), "Versuche über Getreidedruck in Silozellen (Texts on grain pressure in silos)", Zeitschr. d. Vereines deutscher Ingenieure, 39, 1045-1049.

Khezri, E., Mohamad, H., HajiHassani, M. and Fatani, B. (2015), "The stability of shallow circular tunnels in soil considering variations in cohesion with depth”, Tunn. Undergr. Space Technol., 49, 230-240.

Kirsch, A. (2010), "Experimental investigation of the face stability of shallow tunnels in sand", Acta Geotechnica, 5(1), 43-62.

Leca, E. and Dormieux, L. (1990), "Upper and lower bound solutions for the face stability of shallow circular tunnels in frictional material", Geotechnique, 40(4), 581-606.

Mazek, S.A. (2014), "Evaluation of surface displacement equation due to tunnelling in cohesionless soil", Geomech. Eng., Int. J., 7(1), 55-73.

Mollon, G., Dias, D. and Soubra, A.H. (2009), "Probabilistic analysis and design of circular tunnels against face stability", Int. J. Geomech., 9(6), 237-249.

Mollon, G., Dias, D. and Soubra, A.H. (2010), "Face stability analysis of circular tunnels driven by a pressurized shield", J. Geotech. Geoenviron. Eng., 136(1), 215-229.

Mollon, G., Dias, D. and Soubra, A.H. (2011), "Rotational failure mechanisms for the face stability analysis of tunnels driven by a pressurized shield", Int. J. Numer. Anal. Meth. Geomech., 35(12), 1363-1388.

Senent, S. and Jimenez, R. (2015), "A tunnel face failure mechanism for layered ground, considering the possibility of partial collapse”, Tunn. Undergr. Space Technol., 47, 182-192.

Takano, D., Otani, J., Nagatani, H. and Mukunoki, T. (2006), "Application of X-ray CT boundary value problems in geotechnical engineering-Research on tunnel face failure", Proceedings of Geocongress 2006, ASCE, Reston, VA, USA.

Tang, X.W., Liu, W. and Albers, B. (2014), "Upper bound of tunnel face stability in layered soils", Acta Geotechnica, 9(4), 661-671.

$C C$ 


\section{Appendix A}

The upper bound formula derivation and calculation procedures are explained in this section. Fig. 2 shows that any failure mechanism consists of three rigid blocks. To calculate the external and internal dissipated powers, it is necessary to calculate the geometrical properties of these blocks, their cross sections with ground surface and tunnel face and the contact area between each block. Fig. A1 shows the geometry of the first cone which contains the block $B_{1}$ (see Fig. 2). This cone has the axis angle of $\alpha_{1}$ and the diameter of $D_{1}$ which is equal to tunnel's diameter $D$. Therefore, the failure area on tunnel face $A_{T}$, volume of the cone $V_{1}$ and lateral area of this cone $L A_{1}$ can be defined as follows

$$
\begin{gathered}
A_{T}=\frac{\pi D_{1}^{2}}{4} \times \frac{\sqrt{\cos \left(\alpha_{1}-\phi_{2}\right) \cos \left(\alpha_{1}+\phi_{2}\right)}}{\cos \left(\phi_{2}\right)} \\
V_{1}=\frac{\pi D_{1}^{3}}{12} \times \frac{\left(\cos \left(\alpha_{1}-\phi_{2}\right) \cos \left(\alpha_{1}+\phi_{2}\right)\right)^{3 / 2}}{\cos \left(\phi_{2}\right) \sin \left(2 \phi_{2}\right)} \\
L A_{1}=\frac{\pi D_{1}^{2}}{4} \times \cos \left(\alpha_{1}\right) \frac{\sqrt{\cos \left(\alpha_{1}-\phi_{2}\right) \cos \left(\alpha_{1}+\phi_{2}\right)}}{\cos \left(\phi_{2}\right) \sin \left(\phi_{2}\right)}
\end{gathered}
$$

Then the plane $\Delta$ with angle of $m$ with horizontal cuts the first cone leaving an elliptical cross section with diameter $D_{2} . \alpha_{2}$ and $D_{2}$ are defined using the following equations

$$
\alpha_{2}=\left|m-\pi / 2-\alpha_{1}\right|
$$

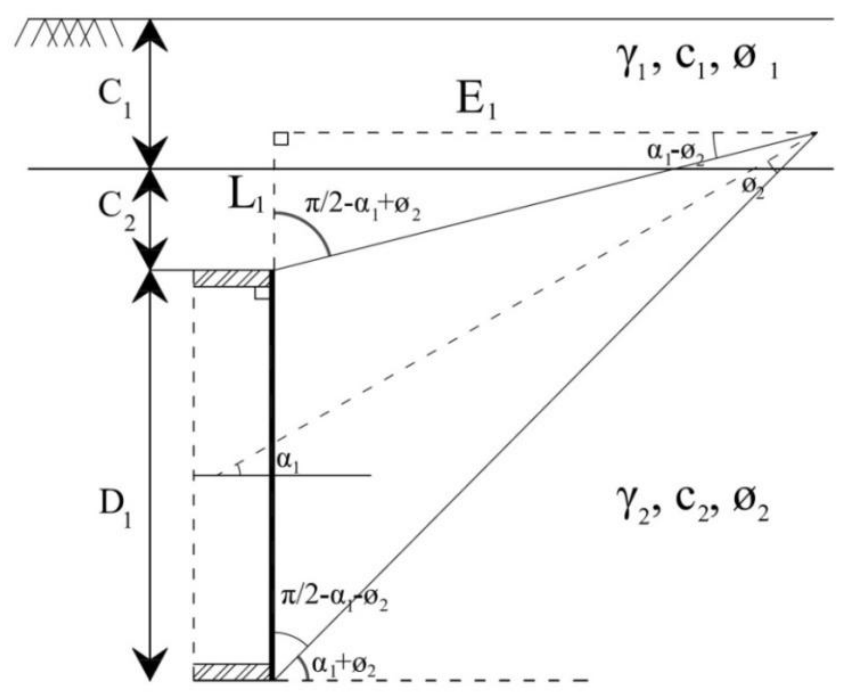

Fig. A1 Position of the first cone in front of the tunnel 


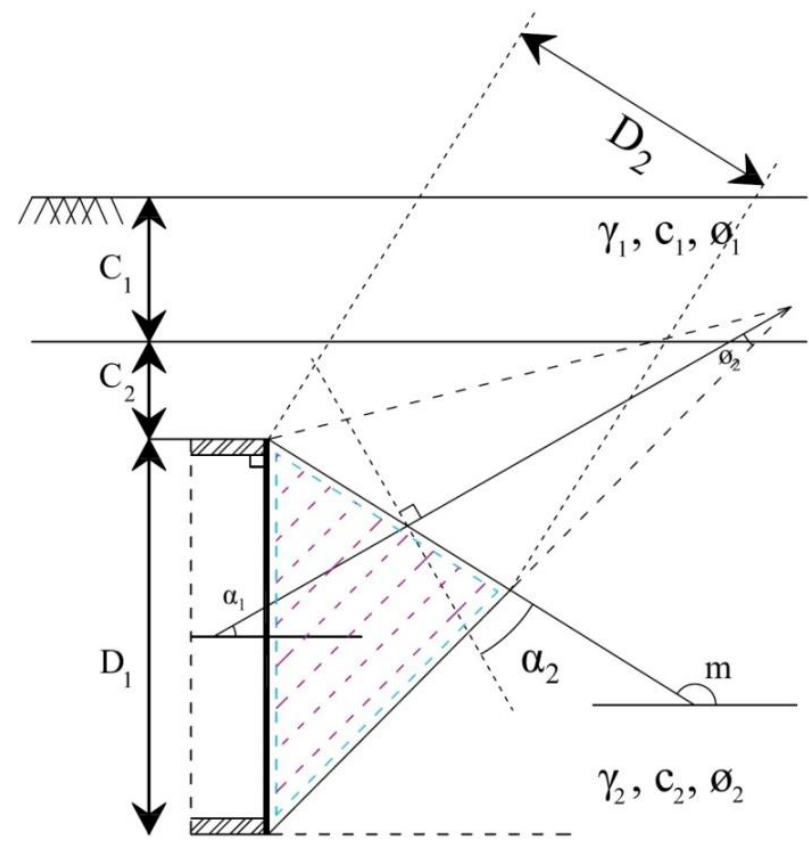

Fig. A2 1 Plane $\Delta$ and sliding block $B_{1}$

$$
D_{2}=\left|\frac{\cos \left(\alpha_{1}+\alpha_{2}\right)}{\sin \left(m-\alpha_{1}+\alpha_{2}\right.}\right|
$$

Adopting $\alpha_{2}$ and $D_{2}$, the cross section of the plane $\Delta$ and the first cone $A_{2}$, the volume $V_{2}$ and lateral area $L A_{2}$ of the cone above the plane $\Delta$ can be defined as follows

$$
\begin{gathered}
A_{2}=\frac{\pi D_{2}^{2}}{4} \times \frac{\sqrt{\cos \left(\alpha_{2}-\phi_{2}\right) \cos \left(\alpha_{2}+\phi_{2}\right)}}{\cos \left(\phi_{2}\right)} \\
V_{2}=\frac{\pi D_{2}^{3}}{12} \times \frac{\left(\cos \left(\alpha_{2}-\phi_{2}\right) \cos \left(\alpha_{2}+\phi_{2}\right)\right)^{3 / 2}}{\cos \left(\phi_{2}\right) \sin \left(2 \phi_{2}\right)} \\
L A_{2}=\frac{\pi D_{2}^{2}}{4} \times \cos \left(\alpha_{2}\right) \frac{\sqrt{\cos \left(\alpha_{2}-\phi_{2}\right) \cos \left(\alpha_{2}+\phi_{2}\right)}}{\cos \left(\phi_{2}\right) \sin \left(\phi_{2}\right)}
\end{gathered}
$$

Inferring to Fig. A2 the lateral area of block $B_{1}, L A_{B 1}$ and volume of block $B_{1}, V_{B 1}$ are

$$
L A_{B 1}=L A_{1}-L A_{2}
$$




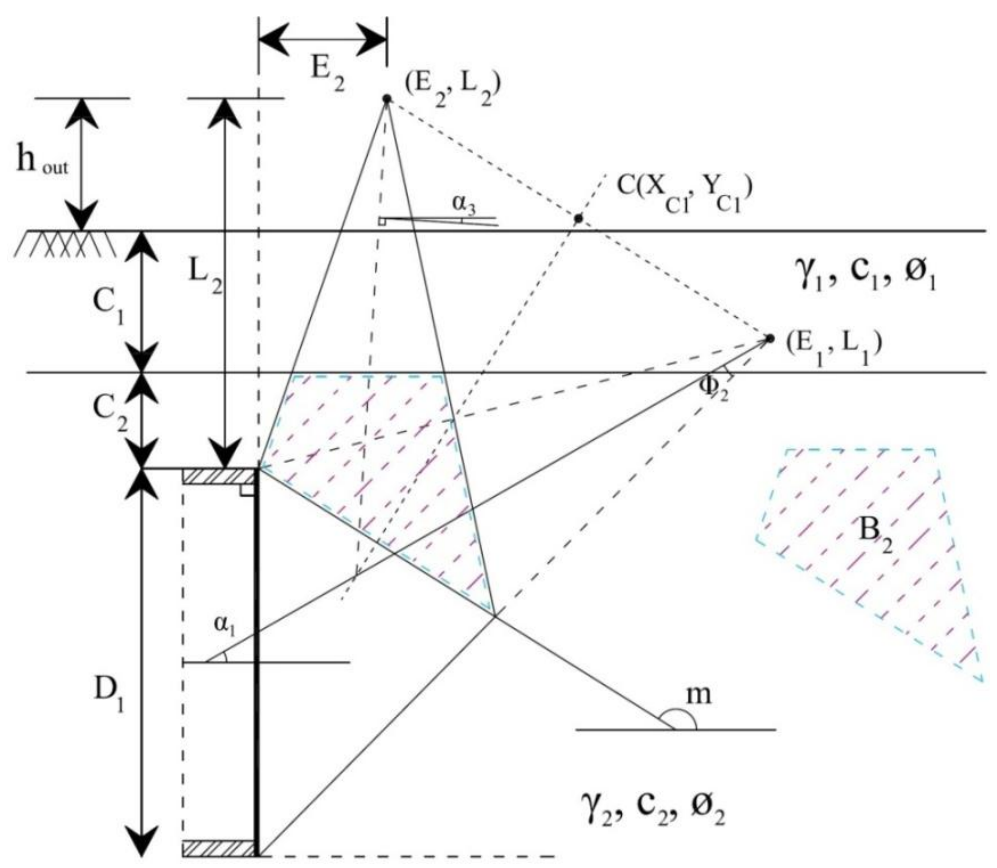

Fig. A3 The position of the apex of the mirrored cone and the sliding block $B_{2}$

$$
V_{B 1}=V_{1}-V_{2}
$$

The second cone which contains the block $B_{2}$ is the mirror image of the eliminated part of the first cone (above the plane $\Delta$ ) with respect to bisector of $A_{2}$. The contact area of blocks $B_{1}$ and $B_{2}$ should be kept constant. The volume $V_{3}$ and the lateral area of the second cone $L A_{3}$ are

$$
\begin{gathered}
V_{3}=V_{2} \\
L A_{3}=L A_{2}
\end{gathered}
$$

The image cone with apex of $\left(E_{2}, L_{2}\right)$ cuts the boundary of layers in an ellipse shape with major diameter of $D_{3}$ (Fig. A3). Its axis makes an angle of $\alpha_{3}$ with normal to the axis. $D_{3}$ and $\alpha_{3}$ parameters can be defined by the following equations where $h_{\text {out } 1}=L_{2}-C_{2}$.

$$
\begin{gathered}
\alpha_{3}=\left|2 m-3 \pi / 2-\alpha_{1}\right| \\
D_{3}=\frac{h_{\text {out } 1} \sin \left(2 \phi_{2}\right)}{\cos \left(\alpha_{3}-\phi_{2}\right) \cdot \cos \left(\alpha_{3}+\phi_{2}\right)}
\end{gathered}
$$

Thus, the cross section of the mechanism with layer's boundary $A_{3}$, the volume $V_{4}$ and lateral area 
$L A_{4}$ of that part of the cone above the layer's boundary can be calculated by Eqs. (A15), (A16) and (A17), respectively. By deducting these parameters from the volume and lateral area of the mirror image cone, the properties of the sliding block $B_{2}$ can be obtained.

$$
\begin{gathered}
A_{3}=\frac{\pi D_{3}^{2}}{4} \times \frac{\sqrt{\cos \left(\alpha_{3}-\phi_{2}\right) \cos \left(\alpha_{3}+\phi_{2}\right)}}{\cos \left(\phi_{2}\right)} \\
V_{4}=\frac{\pi D_{3}^{3}}{12} \times \frac{\left(\cos \left(\alpha_{3}-\phi_{2}\right) \cos \left(\alpha_{3}+\phi_{2}\right)\right)^{3 / 2}}{\cos \left(\phi_{2}\right) \sin \left(2 \phi_{2}\right)} \\
L A_{4}=\frac{\pi D_{3}^{2}}{4} \times \cos \left(\alpha_{3}\right) \frac{\sqrt{\cos \left(\alpha_{3}-\phi_{2}\right) \cos \left(\alpha_{3}+\phi_{2}\right)}}{\cos \left(\phi_{2}\right) \sin \left(\phi_{2}\right)}
\end{gathered}
$$

The volume $V_{B 2}$ and lateral area $L A_{B 2}$ of block $B_{2}$ are

$$
V_{B 2}=V_{3}-V_{4}
$$

$$
L A_{B 2}=L A_{3}-L A_{4}
$$

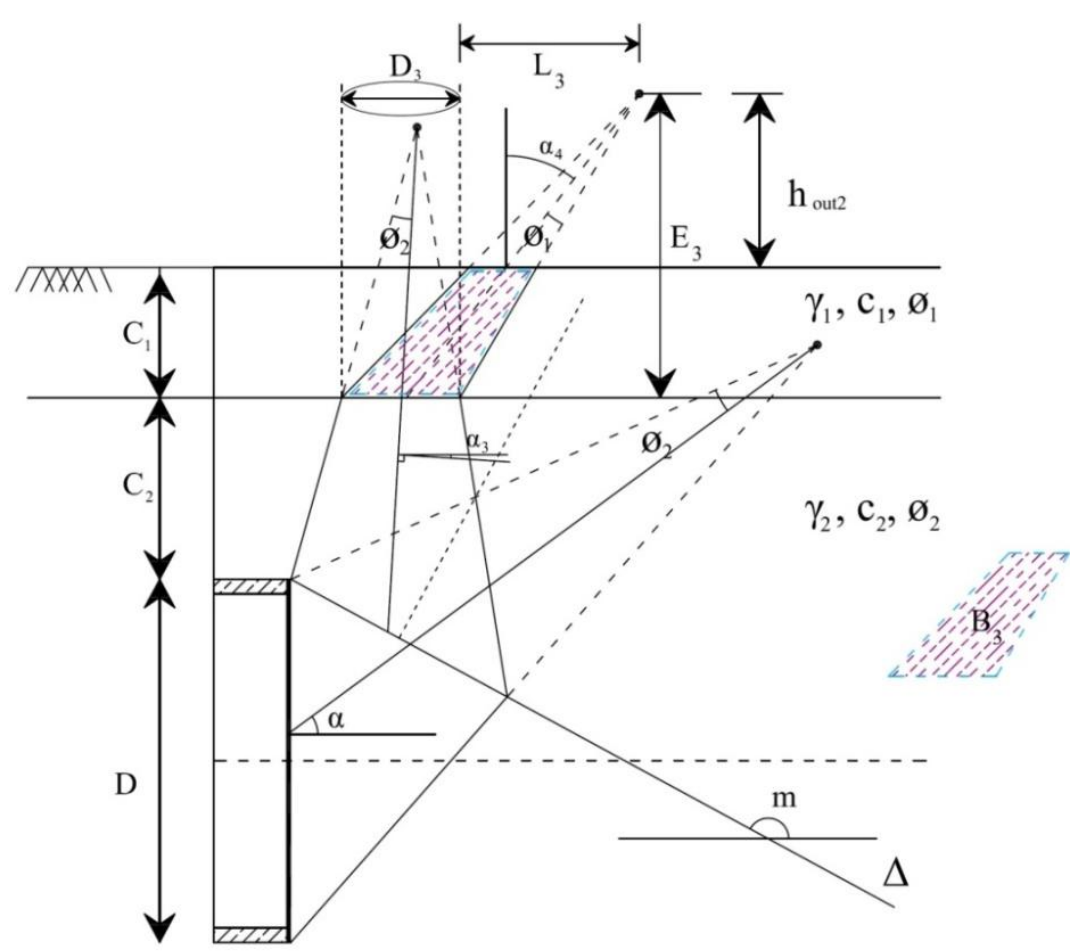

Fig. A4 Formation of the new cone in the upper layer and sliding block $B_{3}$ 
The friction angle of the upper layer $\phi_{1}$ differs from the friction angle of the lower layer $\phi_{2}$. When the mechanism reaches the upper layer, in order to satisfy the normality condition, a new cone with apex angle of the upper layer's friction angle $\left(2 \phi_{1}\right)$ should be formed. The contact area of this new cone (ellipse with diameter of $D_{3}$ ) should be kept the same as the contact area of the cone with lower layers friction angle. Now that we have the diameter $D_{3}$ and apex angle of the new cone $2 \phi_{1}$, the angle of the axis of the new cone $\alpha_{4}$ can then be determined (see Fig. A4).

$$
\alpha_{4}=\cos ^{-1}\left[ \pm \sqrt{\left(\frac{2 b_{1} \cos \left(\phi_{1}\right)}{D_{3}}\right)^{2}+\left(\sin \left(\phi_{1}\right)^{2}\right.}\right]
$$

Thus, the volume $V_{5}$ and lateral area $L A_{5}$ of the new cone (which contains $B_{3}$ ) formed in the upper layer can be defined by the following equations

$$
\begin{gathered}
V_{5}=\frac{\pi D_{3}^{3}}{12} \times \frac{\left(\cos \left(\alpha_{4}-\phi_{2}\right) \cos \left(\alpha_{4}+\phi_{2}\right)\right)^{3 / 2}}{\cos \left(\phi_{2}\right) \sin \left(2 \phi_{2}\right)} \\
L A_{5}=\frac{\pi D_{3}^{2}}{4} \times \cos \left(\alpha_{4}\right) \frac{\sqrt{\cos \left(\alpha_{4}-\phi_{2}\right) \cos \left(\alpha_{4}+\phi_{2}\right)}}{\cos \left(\phi_{2}\right) \sin \left(\phi_{2}\right)}
\end{gathered}
$$

If the mechanism reaches the ground surface, the area of cross section of it with ground surface $A_{6}$, its volume $V_{6}$ and lateral area $\varphi_{6}$ above the ground should be calculated.

$$
\begin{gathered}
A_{6}=\frac{\pi D_{4}^{2}}{4} \times \frac{\sqrt{\cos \left(\alpha_{4}-\phi_{1}\right) \cos \left(\alpha_{4}+\phi_{1}\right)}}{\cos \left(\phi_{1}\right)} \\
V_{6}=\frac{\pi D_{4}^{3}}{12} \times \frac{\left(\cos \left(\alpha_{4}-\phi_{1}\right) \cos \left(\alpha_{4}+\phi_{1}\right)\right)^{3 / 2}}{\cos \left(\phi_{1}\right) \sin \left(2 \phi_{1}\right)} \\
L A_{6}=\frac{\pi D_{4}^{2}}{4} \times \cos \left(\alpha_{4}\right) \frac{\sqrt{\cos \left(\alpha_{4}-\phi_{1}\right) \cos \left(\alpha_{4}+\phi_{1}\right)}}{\cos \left(\phi_{1}\right) \sin \left(\phi_{1}\right)}
\end{gathered}
$$

Where $D_{4}, h_{\text {out } 2}$ and $E_{3}$ are as follow

$$
\begin{gathered}
D_{4}=\frac{h_{\text {out } 2} \times \sin \left(2 \phi_{1}\right)}{\cos \left(\alpha_{4}-\phi_{1}\right) \cos \left(\alpha_{4}+\phi_{1}\right)} \\
h_{\text {out } 2}=E_{3}-C_{1}
\end{gathered}
$$




$$
E_{3}=\frac{D_{3}}{\tan \left(\phi_{1}\right) \times\left[1+\frac{\cos \left(\phi_{1}\right)}{\cos \left(\alpha_{4}\right) \cos \left(\alpha_{4}-\phi_{1}\right)}+\tan \left(\alpha_{4}\right) \times \tan \left(\alpha_{4}+\phi_{1}\right)\right]}
$$

The volume $V_{B 3}$ and lateral area $L A_{B 3}$ of the block $B_{3}$ are

$$
\begin{gathered}
V_{B 3}=V_{5}-V_{6} \\
L A_{B 3}=L A_{5}-L A_{6}
\end{gathered}
$$

The sliding blocks of $B_{1}, B_{2}, B_{3}$ do not move with same velocity and velocity discontinuity occurs in the contact area of the blocks. Therefore a relative velocity of $V_{\mathbf{1 2}}$ between $B_{1}$ and $B_{2}$ and over $\Sigma_{12}$ and another relative velocity $\mathbf{V}_{23}$ between $B_{2}$ and $B_{3}$ and over $\Sigma_{23}$ should be considered. Fig. A5 illustrates the hodographs of velocities for the contact areas. Normality rule imposes that velocities should make angle of $\phi$ with the failure surface. Thus the relationship between the velocities can be written as follows

$$
\begin{gathered}
\mathbf{V}_{1}=\frac{\cos \left(\pi-m-\phi_{1}-\alpha_{3}\right)}{\sin \left(\pi-m-\phi_{1}+\alpha_{1}\right)} \mathbf{V}_{2} \\
\mathbf{V}_{12}=\left[\frac{\cos \left(\pi-m-\phi_{1}-\alpha_{3}\right) \cos \left(\alpha_{1}\right)}{\cos \left(\pi-m-\phi_{1}+\alpha_{1}\right) \cos \left(\pi-m-\phi_{1}\right)}-\frac{\sin \left(\alpha_{3}\right)}{\cos \left(\pi-m-\phi_{1}\right)}\right] \mathbf{V}_{2} \\
\mathbf{V}_{3}=\frac{\cos \left(\alpha_{3}-\phi_{i}\right)}{\cos \left(\alpha_{4}-\phi_{i}\right)} \mathbf{V}_{2} \\
\mathbf{V}_{23}=\frac{-\sin \left(\alpha_{4}-\alpha_{3}\right)}{\cos \left(\phi_{i}-\alpha_{4}\right)} \mathbf{V}_{2}
\end{gathered}
$$

As it is understood from Eqs. (A31) to (A34), all the velocities are translated in term of one velocity $V_{2}$. In the upper bound theorem, the magnitude of velocities is not important as long as the direction and velocities' direction are known. By translating the external and internal powers in terms of one velocity $V_{2}$ and subsequently equating them, the velocity's magnitude can be omitted from the sides of the equation. In Eqs. (A33) and (A34), the index $i$ indicates the layer on which the sliding occurs. Considering the strength parameters of both layers, the shear strength of each soil layer is calculated on the layer's boundary using Eq. (A35). Comparing $\tau_{1}$ and $\tau_{2}$, the smaller $\tau_{i}$ will show on which layer the sliding will occur. It means that the sliding on layer's boundary occurs on the layer with the lower shear strength.

$$
\tau_{i}=c_{i}+\gamma_{1} C_{1} \tan \left(\phi_{i}\right), \quad i=1,2
$$


Plastic energy can be dissipated along the lateral area of each block and sliding surfaces. In general form, the plastic energy per unit area is defined by Eq. (A36).

$$
\frac{d P_{V}}{d \Sigma}=c \mathbf{V} \cdot \cos (\phi)
$$

$L A_{B 1}, L A_{B 2}$ and $L A_{B 3}$ are defined as the lateral areas of the blocks $B_{1}, B_{2}$ and $B_{3}$, respectively. $A_{2}$ is defined as the contact area between the blocks $B_{1}$ and $B_{2}$ and $A_{3}$ is defined as the contact area between the blocks $B_{2}$ and $B_{3}$. Thus the dissipated energy can be written as Eq. (A37).

$$
\begin{aligned}
P_{V} & =P_{V 1}+P_{V 2}+P_{V 3}+P_{V 4}+P_{V 5} \\
& =c_{2} \mathbf{V}_{1} \cos \left(\phi_{2}\right) L A_{B 1}+c_{2} \mathbf{V}_{2} \cos \left(\phi_{2}\right) L A_{B 2} \\
& +c_{1} \mathbf{V}_{\mathbf{3}} \cos \left(\phi_{1}\right) L A_{B 3}+c_{2} \mathbf{V}_{\mathbf{1 2}} \cos \left(\phi_{2}\right) A_{2} \\
& +c_{i} \mathbf{V}_{\mathbf{2 3}} \cos \left(\phi_{2}\right) A_{3}
\end{aligned}
$$

Power of external loads consists of three parameters $P_{T}$ as the power of the minimum support pressure on tunnel face $\left(\sigma_{T}\right), \mathrm{P}_{\mathrm{s}}$ power of surcharge $\left(\sigma_{s}\right)$ and power of soil unit weight $P \gamma$.

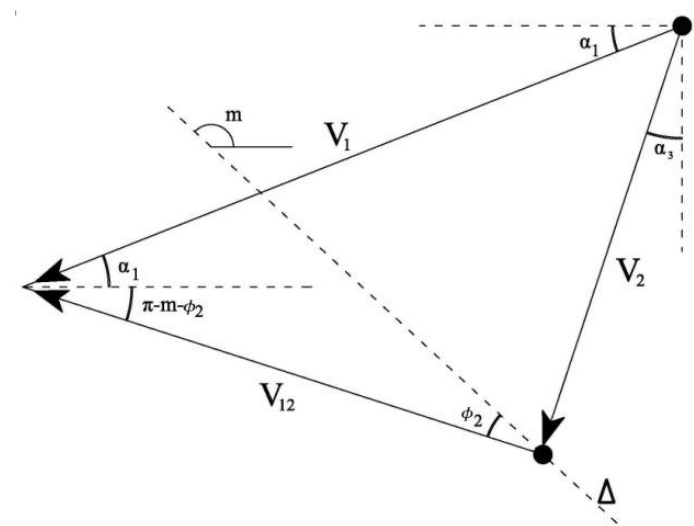

(a) $B_{1}$ and $B_{2}$

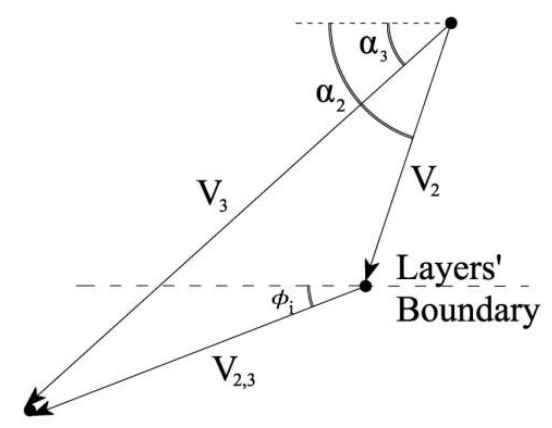

(b) $B_{2}$ and $B_{3}$

Fig. A5 Hodographs of velocity between sliding blocks 


$$
\begin{gathered}
P_{e}=P_{T}+P_{S}+P_{\gamma} \\
P_{T}=-\sigma_{T} \mathbf{V}_{1} \cos \left(\phi_{1}\right) A_{1} \\
P_{\gamma}=\gamma_{2} \mathbf{V}_{\mathbf{1}} \sin (\alpha) V_{B 1}+\gamma_{2} \mathbf{V}_{2} \cos \left(\alpha_{3}\right) V_{B 2}+\gamma_{I} \mathbf{V}_{3} \cos \left(\alpha_{4}\right) V_{B 3} \\
P_{S}=\sigma_{s} \mathbf{V}_{3} \cos \left(\alpha_{4}\right) A_{6}
\end{gathered}
$$

Where $A_{1}$ is the elliptical collapse area of tunnel face, $A_{6}$ is the elliptical (or circular) cross section of mechanism with ground surface, $V_{B 1}, V_{B 2}$ and $V_{B 3}$ are the volumes of the sliding blocks $B_{1}, B_{2}$ and $B_{3}$, respectively. Equating $P_{e}=P_{V}$ for every possible combination of $\alpha$ and $m$, results in the minimum support pressure $\sigma_{T}$. Maximum of these $\sigma_{T}$ values corresponds to the estimated minimum support pressure.

To find the optimum results for the mechanism, one should begin by finding the optimum values of $\alpha$ and $m$ in Fig 2. To find the optimum values of minimum support pressure, for every possible value of $\alpha, 0^{\circ}$ to $\left(\pi / 2-\phi_{1}\right)$, the angle of $m$ would vary from $(\pi / 2+\alpha)$ to $(3 \pi+2 \alpha) / 4$. For all possible combinations of $\alpha$ and $m$, the minimum support pressures are calculated and the maximum value of $\sigma_{T}$ is reported as the answer of the problem. 